Check for updates

Cite this: RSC Adv., 2017, 7, 21901

\title{
Role of hydrophilic groups in acid intercalated graphene oxide as a superionic conductor
}

Received 9th February 2017

Accepted 5th April 2017

DOI: $10.1039 / \mathrm{c} 7 \mathrm{ra01634d}$

rsc.li/rsc-advances
Kosuke Wakata, ${ }^{a}$ Md. Saidul Islam, ${ }^{a}$ Mohammad Razaul Karim, ${ }^{\text {ab }}$ Kazuto Hatakeyama, ${ }^{a}$ Nurun Nahar Rabin, ${ }^{a}$ Ryo Ohtani, (D) a Masaaki Nakamura, ${ }^{a}$ Michio Koinuma ${ }^{a}$ and Shinya Hayami (D) *ac

Hybrid materials that are obtained from intercalation of different polar and hydrophilic acid guest molecules within graphene oxide (GO) have been found to exhibit high proton conductivity. The accommodation of these guest precursors within the GO interlayer takes place through weak physical forces. PXRD patterns with enhanced interlayer distance confirmed successful intercalation processes. Raman and IR spectral data reveal the absence of any covalent bond between the functional groups of the guest and the GO host. TGA data confirms improved water adsorbing capacity of the hybrids. At ambient conditions and $90 \%$ relative humidity $(\mathrm{RH})$, a $\mathrm{RH}$ dependent impedance study shows that intercalation of formic acid (FA) and phosphoric acid (PA) within GO results in $\sim 7$ times higher proton conductivity compared with that for the pristine GO sample. The low activation energy values suggest that proton conduction in the samples is aided by the Grotthuss mechanism. Improvement in the water adsorbing capacity is primarily responsible for such high proton conductivity. The current study suggests that cheap and environmentally friendly GO-based intercalated hybrid materials, such as GO/PA and GO/FA with enhanced proton conductivity, can be appropriate replacements for the expensive Nafion-based solid electrolytes.

\section{Introduction}

Efficient solid electrolyte for proton exchange is an essential component in fuel cells, sensors, chemical filters and biological transport systems. In fact, the efficiencies or sensitivities of numerous energy generating systems largely depend on fast ionic movement. ${ }^{1}$ Stable, non-toxic, easily processable and inexpensive carbon based proton conductive solid electrolytes have drawn recent interest as replacements for the commercial Nafion polymer electrolyte, which has significant limitations in terms of high-cost. ${ }^{2}$ Among various oxidized forms of carbon materials including fullerene, carbon nanotubes, amorphous carbon and graphene, the 2D shaped graphene oxide (GO) has attracted considerable attention because GO's shapes and chemical structures reveal the best fit for the proton conduction process. ${ }^{3}$ Due to the oxidation of graphite, the GO surface becomes functionalized by various oxygen-containing groups, predominantly hydroxy and epoxy moieties, on both its basal planes and edges. These structures, in their humidified form,

${ }^{a}$ Department of Chemistry, Graduate School of Science and Technology, Kumamoto University, 2-39-1 Kurokami, Chuo-ku, Kumamoto 860-8555, Japan. E-mail: hayami@kumamoto-u.ac.jp

${ }^{b}$ Department of Chemistry, School of Physical Sciences, Shahjalal University of Science and Technology, Sylhet-3114, Bangladesh

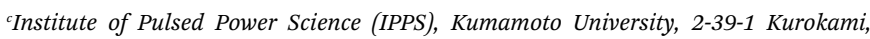
Chuo-ku, Kumamoto 860-8555, Japan can support super first proton transportation through reformation of hydrogen bonds. The possibility of GO based solid electrolyte was first reported by Raidongia et al., who presented the ionic movement through GO interlayers in aqueous solution. ${ }^{4}$ Later, several attempts were made to enhance the proton conductivity of GO based materials. Some typical enhancements include the development of GO-Nafion hybrid, GOpolydopamine hybrid and GO-sulfonated derivatives. ${ }^{5}$ High inplane proton conductivity was observed in single layer GO nanosheet. ${ }^{6}$ This in-plane conductivity was increased further by increasing the oxygen content or by applying multilayer stacking of the GO nanosheet. ${ }^{7}$ According to these reports, retention of epoxy and hydroxyl/carboxyl groups is necessary to improve water adsorbing capacity and flexibility of the conduction channels. These are the key factors to enhance proton conductivity. Decrease in proton conductivity is due to blocking of the epoxy sites by metal ions or by the insertion of bulky hydrophilic groups into GO moiety. ${ }^{8}$ Accordingly, in a recent study we observed the dependency of proton conductivity of oxidized graphitic materials, on both the oxygen content and the interlayer distance. ${ }^{9}$ Intercalating hydrophilic polar components into the GO interlayer has been proven to improve water adsorbing capacity, expansion of interlayer distance and the construction of a more ordered hydrogen bonded network for easier proton hopping. Based on this fact, in this study, we have intercalated organic and inorganic acid molecules within 
the GO interlayer to generate various GO-hybrids. The proton conductivity of the as-prepared $\mathrm{GO}-\mathrm{HNO}_{3}(\mathrm{GO} / \mathrm{NA}), \mathrm{GO}-\mathrm{HCl}$ (GO/HA), GO- $\mathrm{H}_{3} \mathrm{PO}_{4}$ (GO/PA), GO-HCOOH (GO/FA) and GO$\mathrm{CH}_{3}-\mathrm{COOH}(\mathrm{GO} / \mathrm{AA})$ reveals the influence of intercalated groups on proton transportation. In addition, the $\mathrm{GO}-\mathrm{NH}_{3}$ (GO/AM) hybrid was considered for further understanding the role of intercalating groups for proton conduction. It was found that without forming any chemical bonds, the GO-hybrids stabilize through weak van der Waals forces between the intercalators and GO. This physical binding results in very high proton conductivity. Particularly, GO/FA showed highest proton conductivity $\left(1.7 \times 10^{-2} \mathrm{~S} \mathrm{~cm}^{-1}\right)$, which was almost 7 -fold higher than that in the similar GO sample $\left(2.4 \times 10^{-3} \mathrm{~S} \mathrm{~cm}^{-1}\right)$. Therefore, we propose that GO hybrids with enhanced water adsorbing capability could be a better replacement of commercially available proton conductors.

\section{Experimental}

\subsection{Preparation of GO}

Modified Hummers' method was used to prepare GO from bulk graphite powder. ${ }^{10}$ A $1000 \mathrm{~mL}$ beaker containing $1.0 \mathrm{~g}$ graphite powder was placed in an ice bath. Furthermore, $48 \mathrm{~mL} \mathrm{H}_{2} \mathrm{SO}_{4}$ (97\%) and $1.0 \mathrm{~g}$ of $\mathrm{NaNO}_{3}$ was carefully added and mixed homogeneously using a magnetic stirrer for $30 \mathrm{~min}$. Then, $3.0 \mathrm{~g}$ $\mathrm{KMnO}_{4}$ powder was added to the mixture. The temperature of the mixture was kept below $20^{\circ} \mathrm{C}$ for $1 \mathrm{~h}$ in a vigorously stirring condition. The temperature of the mixture was then increased to $35{ }^{\circ} \mathrm{C}$ for $30 \mathrm{~min}$. Subsequently, $180 \mathrm{~mL}$ water was slowly added to the mixture, and this was followed by the temperature being raised to $95{ }^{\circ} \mathrm{C}$ for another $2 \mathrm{~h}$. Finally, $400 \mathrm{~mL}$ water and $12 \mathrm{~mL} \mathrm{H}_{2} \mathrm{O}_{2}$ solution (30\%) was added. The mixture was centrifuged (4000 rpm, $20 \mathrm{~min}$ ) and the precipitate was washed with $5 \% \mathrm{HCl}$ solution once and with deionised water three times. The precipitate was dried at $60{ }^{\circ} \mathrm{C}$ for $24 \mathrm{~h}$. It was then dispersed in water $\left(0.8 \mathrm{~g} \mathrm{~L}^{-1}\right)$ under sonication for 2 hours, and this was followed by discarding the aggregated carbon through centrifugation at $4000 \mathrm{rpm}$ for 1 hour.

\subsection{Preparation of GO-hybrid}

GO-hybrids were prepared by mixing GO dispersion $\left(0.8 \mathrm{~g} \mathrm{~L}^{-1}\right)$ with intercalators in a weight ratio of intercalators : GO as $1: 0.08$. The mixing was aided by mild ultrasonication. In a typical method, $10 \mathrm{~mL}$ GO dispersion $\left(0.8 \mathrm{~g} \mathrm{~L}^{-1}\right)$ was mixed with $0.12 \mathrm{~mL}\left(0.26 \mathrm{~mol} \mathrm{~L}^{-1}\right) \mathrm{FA}, 0.21 \mathrm{~mL}\left(0.15 \mathrm{~mol} \mathrm{~L}^{-1}\right) \mathrm{PA}, 0.1$ $\mathrm{mL}\left(0.12 \mathrm{~mol} \mathrm{~L}^{-1}\right) \mathrm{HA}, 0.23 \mathrm{~mL}\left(0.15 \mathrm{~mol} \mathrm{~L}^{-1}\right) \mathrm{NA}, 0.17 \mathrm{~mL}$ $\left(0.17 \mathrm{~mol} \mathrm{~L}^{-1}\right) \mathrm{AA}$ and $0.5 \mathrm{~mL}\left(0.12 \mathrm{~mol} \mathrm{~L}^{-1}\right) \mathrm{AM}$ in order to prepare GO/FA, GO/PA, GO/HA, GO/NA, GO/AA and GO/AM respectively. We prepared different composites with different compositions of GO and acid. However, we found that hybrids made from the as-mentioned ratio in general, revealed stable formation of hybrids and optimum conductivity. GO and GO/ hybrid papers were prepared by filtering $10 \mathrm{~mL}$ of the respective solutions using a membrane filter $(0.4 \mu \mathrm{m}$ pore size, Merck Millipore) under reduced pressure and successive vacuum drying. During filtration, the paper was made by stacking the nanosheets over each other. The paper needed not to be calendared by applying any external pressure in order to control the thickness. For GO/comb electrode fabrication, a combshaped gold electrode was soaked with one drop $(0.15 \mu \mathrm{L})$ of dilute GO dispersion, followed by vacuum drying for $1 \mathrm{~h}$. GO-hybrid comb electrodes also were prepared by the same procedure.

\subsection{Proton conductivity measurement}

The proton conductivities of the GO and GO-hybrids were measured by the four probe AC method, using an impedance/ gain phase analyzer (Solartron 1260) over the frequency range from 1 to $10^{6} \mathrm{~Hz}$. A very high $\left(>10^{8} \Omega\right)$ resistance of the bare comb electrode confirmed the absence of any parasitic impedance contribution. Proton conductivity $(\sigma)$ was calculated according to $\sigma=(1 / R) \times(d / A)$ where, $R, d$ and $A$ represent the resistance (radius of the semicircular curve of the Nyquist plot), distance between two combs and the area of the samples.

\section{Results and discussion}

Characterizations of GO and GO-hybrids confirm the presence of intercalated molecules at the GO interlayer (Fig. 1). Fig. 1a shows the AFM images of GO and GO/PA, where the GO surface seems to be smoother than that of GO/PA. This difference in surface morphology arises from the presence of $\mathrm{PO}_{4}{ }^{3-}$ or other related products at the interlayer. ${ }^{11}$ No shift in the band (D and G) position or band intensity ratio in Raman spectra (Fig. 1b) of GO and GO/PA confirms the absence of chemical bonds between GO and intercalated molecules.
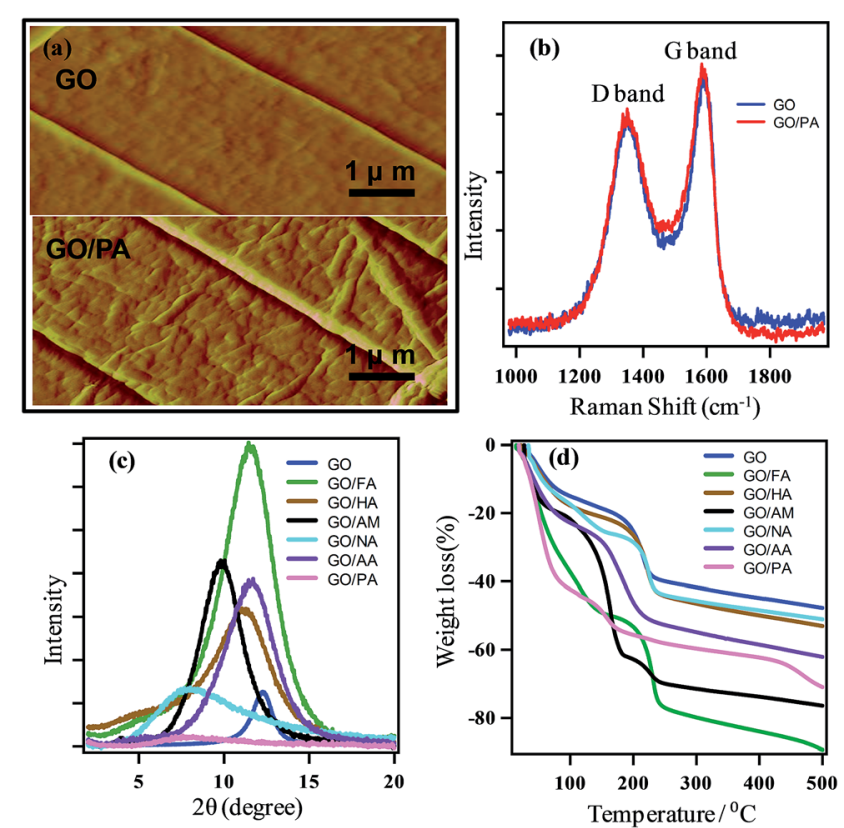

Fig. 1 Characterization of GO and GO-hybrids. (a) AFM image of GO and GO/PA, (b) Raman spectra of GO and GO/PA, (c) powder XRD pattern of GO and GO-hybrids and (d) TGA data of GO and GO-hybrid after $2 \mathrm{~h}$ incubation at $80 \% \mathrm{RH}$. 
The PXRD patterns of GO and GO-hybrids are shown in Fig. 1c. The interlayer distance of GO is calculated to be $7.7 \AA$, whereas all GO-hybrid samples showed a broad peak at the lower $2 \theta$ angle, associated with the increase in the interlayer distance (Table 1). The increment in the interlayer distance indicates the presence of bulky intercalated ions. ${ }^{12}$ Maximum interlayer distance of $11.3 \AA$ was observed for GO/PA. TGA of GO and GO-hybrid samples after $2 \mathrm{~h}$ incubation at $80 \%$ relative humidity (RH) are shown in Fig. 1d. For all samples, the weight losses up to a temperature of $100{ }^{\circ} \mathrm{C}$ imply the presence of adsorbed (inter-lamellar) water molecules. The quantity of weight loss at $100{ }^{\circ} \mathrm{C}$ is presented in Table 1 . At $80 \% \mathrm{RH}$, the amount of water adsorbed by GO-hybrids is much higher than that for GO. Among the GO hybrids, GO/PA possesses the highest water uptaking capacity as $42.35 \%$. This value is almost 2.9 times higher than that for GO. At $100-200^{\circ} \mathrm{C}$, the weight loss represents the decomposition of functional groups. Compared with GO, higher weight losses for all GO hybrids at high temperatures also indicate the presence of excess intercalated water molecules in the hybrids.

IR spectra in Fig. 2 show the presence of various functional groups in GO and GO hybrids. Characteristic peaks for GO at 1720,1635 and $1050 \mathrm{~cm}^{-1}$ indicate the stretching frequencies of $-\mathrm{C}=\mathrm{O}$ (carboxyl and carbonyl), $-\mathrm{COH}$ and $-\mathrm{C}-\mathrm{O}-\mathrm{C}-$ functional groups. The broad intense band at $3350 \mathrm{~cm}^{-1}$ indicates the presence of hydroxyl groups. Retention of all peaks after intercalation confirms the presence of oxygenated sites in GO-

Table 1 Interlayer distance and $\%$ weight loss at $100^{\circ} \mathrm{C}$ of GO and GO hybrids

\begin{tabular}{lll}
\hline $\begin{array}{l}\text { Sample } \\
\text { name }\end{array}$ & $\begin{array}{l}\text { Interlayer } \\
\text { distance }\end{array}$ & $\begin{array}{l}\text { \% weight } \\
\text { loss at } 100{ }^{\circ} \mathrm{C}\end{array}$ \\
\hline $\mathrm{GO}$ & $7.7 \AA$ & 15.03 \\
$\mathrm{GO} / \mathrm{FA}$ & $7.9 \AA$ & 37.35 \\
$\mathrm{GO} / \mathrm{HA}$ & $8.0 \AA$ & 17.76 \\
$\mathrm{GO} / \mathrm{AM}$ & $8.9 \AA$ & 22.81 \\
$\mathrm{GO} / \mathrm{AA}$ & $7.9 \AA$ & 22.84 \\
GO/PA & $11.3 \AA$ & 42.35 \\
GO/NA & $11.2 \AA$ & 17.45
\end{tabular}

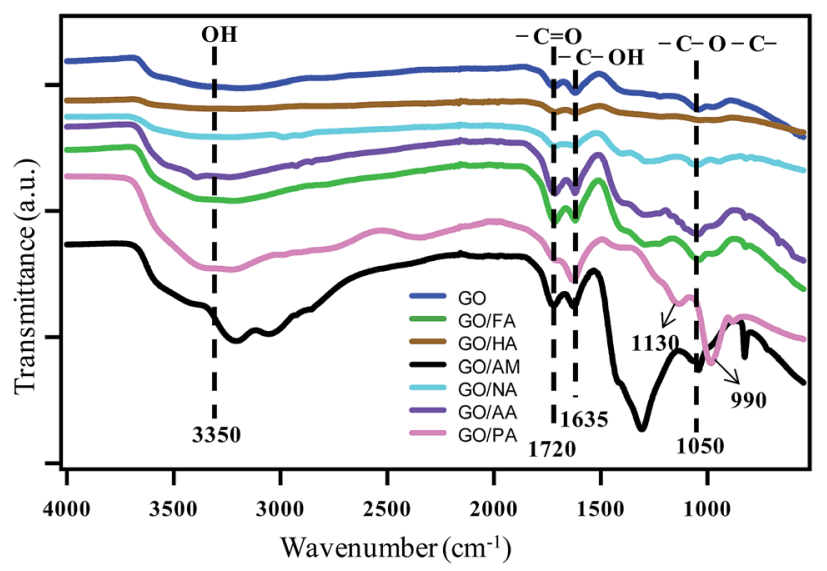

Fig. 2 IR spectra of GO and GO-hybrids. hybrids, which are consistent with previous reports. ${ }^{13}$ In addition, GO-hybrids show the presence of characteristic peaks for some other groups, which are absent in GO. This observation indicates the existence of intercalated species in the hybrids. For example in GO/PA, strong peaks at $990 \mathrm{~cm}^{-1}$ and $1130 \mathrm{~cm}^{-1}$ for $\mathrm{P}-\mathrm{O}$ stretching can be assigned for the existence of the $\mathrm{PO}_{4}{ }^{3-}$ group. ${ }^{14}$

Results for proton conductivity measurements of GO and GO-hybrids are shown in Fig. 3. The Nyquist plots (Fig. 3a) obtained from fitting the real $\left(\mathrm{Z}^{\prime}\right)$ and imaginary parts $\left(\mathrm{Z}^{\prime \prime}\right)$ of impedance for GO/FA at $30^{\circ} \mathrm{C}$ and $30-90 \% \mathrm{RH}$ have been found to take the shape of distorted semicircular curves with the appearance of a second circle. This pattern indicates a proton driven conductivity. The proton-oriented conductivity was further confirmed from isotopic effects. ${ }^{15} \mathrm{RH}$-dependent proton conductivity of GO and GO-hybrids at room temperature is shown in Fig. 3b. Proton conduction of all samples increases with respect to RH. It has been found that GO/FA and GO/PA show higher conductivity than that for GO, whereas the values slightly decrease for GO/HA, GO/NA and GO/AA. The conductivity is much lower for GO/AM. The highest value of proton conductivity is displayed by GO/FA. The conductivities of GO and GO/FA are increased from $9.4 \times 10^{-7}$ and $9.7 \times 10^{-6} \mathrm{~S} \mathrm{~cm}^{-1}$ at $40 \% \mathrm{RH}$ to $2.4 \times 10^{-3}$ and $1.7 \times 10^{-2} \mathrm{~S} \mathrm{~cm}^{-1}$ at $90 \% \mathrm{RH}$, respectively. At room temperature and $90 \% \mathrm{RH}$, proton conductivity of GO/PA, GO/NA, GO/HA, GO/AA and GO/AM are $1.1 \times 10^{-2}, 2.1 \times 10^{-3}, 2.3 \times 10^{-3}, 1.9 \times 10^{-3}$ and $8.4 \times 10^{-4} \mathrm{~S}$ $\mathrm{cm}^{-1}$ respectively. Proton conduction within the as-prepared hybrids is in the order of GO/FA > GO/PA >> GO > GO/HA > GO/NA > GO/AA > GO/AM. Table 2 presents the proton conductivity of GO and GO hybrids at R.T and $90 \% \mathrm{RH}$.
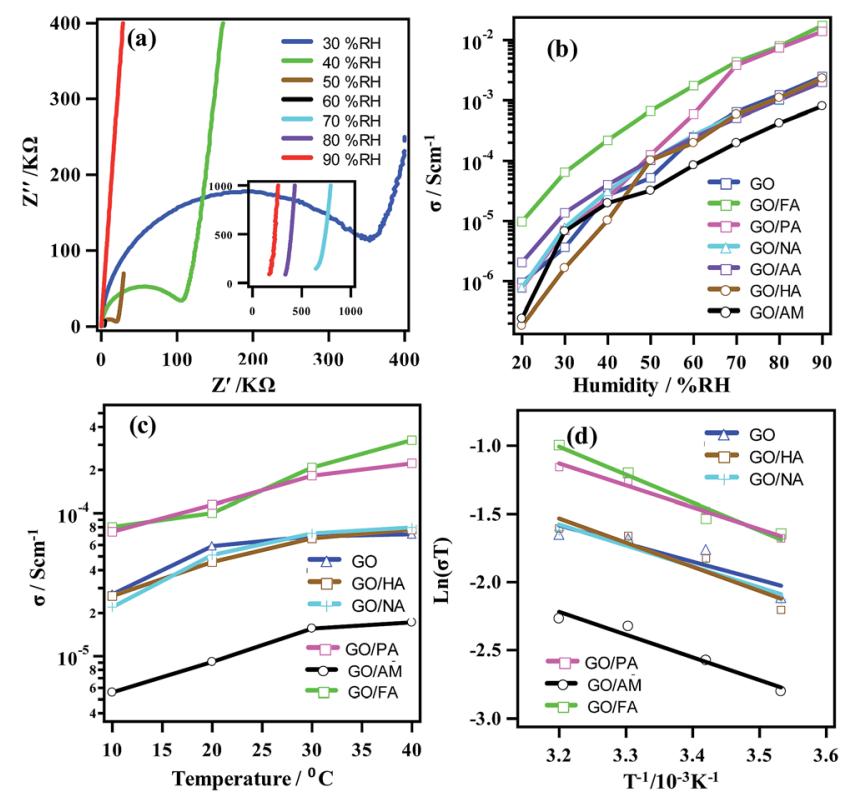

Fig. 3 Proton conductivity measurements of $\mathrm{GO}$ and GO-hybrids. Nyquist plots for GO/FA hybrid (a); proton conductivity of the GO and $\mathrm{GO}$-hybrids with respect to $\mathrm{RH}$ at RT (b); temperature-dependent conductivities of the GO and GO-hybrids at $60 \% \mathrm{RH}$ (c) and Arrhenius plot for $\ln (\sigma T)$ vs. $T^{-1}$ for activation energy (d). 
Table 2 Proton conductivity and activation energy of GO and GO hybrids

\begin{tabular}{lll}
\hline $\begin{array}{l}\text { Sample } \\
\text { name }\end{array}$ & $\begin{array}{l}\text { Proton conductivity } \\
\text { at } 25{ }^{\circ} \mathrm{C} \text { and } 90 \mathrm{RH}\left(\mathrm{S} \mathrm{cm}^{-1}\right)\end{array}$ & $\begin{array}{l}\text { Activation energy } \\
E_{\mathrm{a}}(\mathrm{eV})\end{array}$ \\
\hline $\mathrm{GO}$ & $2.4 \times 10^{-3}$ & 0.12 \\
$\mathrm{GO} / \mathrm{FA}$ & $1.7 \times 10^{-2}$ & 0.14 \\
$\mathrm{GO} / \mathrm{HA}$ & $2.3 \times 10^{-3}$ & 0.15 \\
$\mathrm{GO} / \mathrm{AM}$ & $8.4 \times 10^{-4}$ & 0.17 \\
$\mathrm{GO} / \mathrm{AA}$ & $1.9 \times 10^{-3}$ & 0.13 \\
$\mathrm{GO} / \mathrm{PA}$ & $1.1 \times 10^{-2}$ & 0.13 \\
GO/NA & $2.1 \times 10^{-3}$ & 0.15 \\
& &
\end{tabular}

Fig. $3 \mathrm{c}$ shows the temperature dependent proton conductivity at $60 \% \mathrm{RH}$.

An increase in conductivity values with temperature was observed for all samples. At $10{ }^{\circ} \mathrm{C}$ and $60 \% \mathrm{RH}$, the $\sigma$ values for GO and GO/FA were found to be $2.7 \times 10^{-5}$ and $8.1 \times 10^{-5} \mathrm{~S}$ $\mathrm{cm}^{-1}$, respectively; the conductivities at $40{ }^{\circ} \mathrm{C}$ reached $7.1 \times$ $10^{-4}$ and $3.4 \times 10^{-4} \mathrm{~S} \mathrm{~cm}^{-1}$, respectively. Activation energies $\left(E_{\mathrm{a}}\right)$ of proton conduction through GO and GO-hybrids have been measured from the Arrhenius plots of $\ln (\sigma T)$ versus $T^{-1}$ (Fig. 3d). Respective activation energy values are presented in Table 2. Low $E_{\mathrm{a}}$ values $(0.12-0.17 \mathrm{eV})$ of GO and GO-hybrids suggest that all the samples conduct protons through the Grotthuss mechanism. Moreover, insignificant differences in $E_{\mathrm{a}}$ among GO and GO-hybrids revel that their proton conduction mechanisms does not change due to intercalation.

Although it is expected that intercalation with polar hydrophilic groups on the GO interlayer will increase GO's proton conductivity, it is observed that only PA and FA can increase the proton conductivity while locating within the GO layers. Intercalation of NA, HA and AA causes a slight decrease in the proton conductivity. Compared with GO, the high proton conductivity of GO/PA and GO/FA can be explained in terms of (i) increase in water adsorbing capacity and (ii) hydration behaviour and hydration dynamics inside the GO walled channels. Proton conductivity is directly related to the amount of adsorbed water. The extent of water content in GO, GO/PA, GO/FA at $80 \% \mathrm{RH}$ (Fig. 1d) indicates a significant improvement of the water adsorbing capacity due to the intercalation of PA and FA. Compared with GO, about 2.8 and 2.5 times higher water content is observed in GO/PA and GO/FA, respectively. The hydrophilic sites $\left(\mathrm{PO}_{4}{ }^{3-}, \mathrm{COOH}\right)$ of the intercalated precursors have very high capability for fixing water molecules. Therefore, in addition to the oxygenated sites in GO, the hydrophilic groups of intercalated molecules support the hydrogen bond reformation process in a faster manner in between the humidified layers of GO/PA and GO/FA. Moreover, Powder XRD patterns in Fig. 1c show the expansion of the interlayers due to the insertion of intercalated molecules between two GO walls (Scheme 1a and b). Previously, we also found that sulfate groups and $\gamma$-hydroxy sulphonic acid groups are chemically inactive towards GO. ${ }^{11,16}$ The hydrophilic sites of both the GO and intercalators remain unchanged in GO/PA and GO/FA (Fig. 2) while being stabilized by weak forces. This type of stabilization is technically desirable as chemical bond formation diminishes
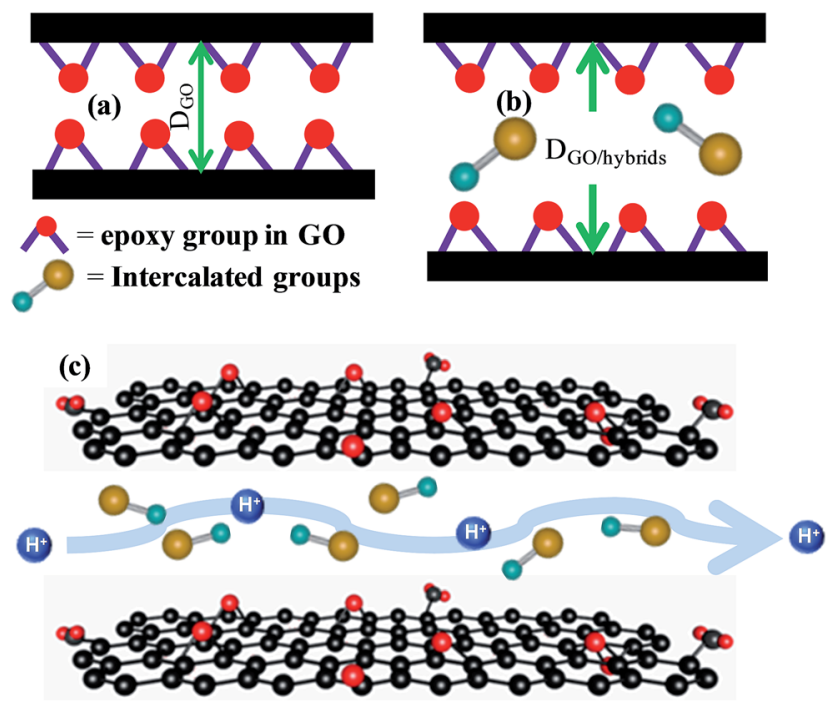

Scheme 1 Schematic for the distance between two GO walls in GO (a) and GO-hybrid (b). Intercalated molecules are randomly distributed at the GO interlayer, which is responsible for the increasing interlayer distance. (C) Proposed proton conduction pathway through the GOhybrid.

the fundamental properties of GO by blocking epoxy and hydroxyl groups. The amount of hydrophilic sites in GO/PA and $\mathrm{GO} / \mathrm{FA}$ are higher due to the presence of intercalated groups at the interlayer. Therefore, PA and FA intercalation not only increase the oxygenated sites and hydrophilicity of GO, but also attribute flexibility to the GO interlayer. It seems that the reformation of hydrogen bonds is not affected by the presence of intercalated molecules; in contrast the generated flexible cavity can enhance the conductivity (Scheme 1c). The low $E_{\text {a }}$ values reveal the occurrence of the 'Grotthuss mechanism' for proton conduction through the GO-hybrids, indicating a possibility of the materials being used for practical application. Intercalation of HA, NA, AA within the GO interlayer was associated with an increase in interlayer distance, but it failed to improve GO's proton conductivity. Two major facts are deemed to be responsible for this contradictory issue. First, through the intercalation of acid molecules, favourable polar sites in the GO interlayer are generated. However, there exists a possibility of negatively charged oxygenated sites in GO being masked due to their attachment with the $\mathrm{H}^{+}$ions dissociated from the acid molecules. This fact can result in the lowering of water uptake capacity. ${ }^{6}$ Second, bulky hydrophobic parts connected to hydrophilic sites can create a barrier in the proton conduction channel. We propose that both these issues are associated with the dissociation constant of the acid molecules and the number of carbons in the chains. However, more precise investigations and findings are beyond our experimental capacity at this moment. IR spectra confirm the decrease in the number of oxygen functional sites in the case of GO/NA and GO/HA. TGA data also implies that compared with GO/PA or GO/FA, the water adsorbing capacity of $\mathrm{HO} / \mathrm{HA}$ and GO/NA is lower. For GO/ $\mathrm{AA}$, we propose that dissociation of the acid molecules at humidified conditions plays the major role for the loss in 
conductivity. In order to clarify this fact, we also studied an AM intercalated GO hybrid.

The GO/AM hybrid displays a proton conductivity 2.8 times lower than that of GO. The XRD pattern implies that the interlayer distance increases, and the retention of the oxygen functional groups is confirmed is from the IR analysis. However, the low conductivity herein also results from the blocking of the oxygenated sites by the $\mathrm{H}^{+}$ion. It is clear that designing an efficient GO based proton conducting solid electrolyte demands very specific conditions to be satisfied, which include improvement of water adsorbing capacity, increase in the interlayer distance and ensuring a flexible channel to support faster reformation of hydrogen bonds. Since all these prerequisite conditions are satisfied by GO/PA and GO/FA, they exhibit the highest conductivities.

\section{Conclusions}

GO-hybrids have been prepared successfully by intercalating hydrophilic acid molecules into the GO interlayer. PXRD data indicates that interlayer distance increases as the intercalated hydrophilic molecules locate mainly within the faces of GO. IR and RAMAN data reveal that the intercalated groups become attached to the GO nanosheet without forming any chemical bond. In contrast, weak forces such as van der Waals attractions and $\pi-\pi$ stacking seem to play the key role behind the stability. Proton conduction within the as prepared hybrids is in the following order: $\mathrm{GO} / \mathrm{FA}>\mathrm{GO} / \mathrm{PA}>>\mathrm{GO}>\mathrm{GO} / \mathrm{HA}>\mathrm{GO} / \mathrm{NA}$ $>\mathrm{GO} / \mathrm{AA}>>\mathrm{GO} / \mathrm{AM}$. GO/FA and GO/PA show an almost 7-fold higher proton conductivity than that of similar GO samples. The higher degree of adsorbed water due to the presence of hydrophilic sites at the intercalated acid molecules is responsible for offering an increased number of proton hopping sites, which is the major factor for the increase in proton conductivity of GO/FA and GO/PA. Moreover, low activation energy associated with proton conduction implies that practical application will be a possibility in near future. The low conductivity of GO/HA, GO/NA, GO/AA, GO/AM, GO/FA, GO/PA seems to result from the blocking of GO's hydrophilic sites by the $\mathrm{H}^{+}$ion, generated from the dissociation of acid molecules in humidified conditions.

\section{Acknowledgements}

This study was supported by KAKENHI Grant-in-Aid for Scientific Research (B) 26288026 JSPS, Japan.

\section{Notes and references}

1 G. Alberti and M. Casciola, Solid State Ionics, 2001, 145, 3; G. A. Voth, Acc. Chem. Res., 2006, 39, 143; K. D. Kreuer, Chem. Mater., 1996, 8, 610; M. Armand, F. Endres, D. R. MacFarlane, H. Ohno and B. Scrosati, Nat. Mater., 2009, 8, 621.
2 S. J. Paddison, Annu. Rev. Mater. Res., 2003, 33, 289; S. J. Peighambardoust, S. Rowshanzamir and M. Amjadi, Int. J. Hydrogen Energy, 2010, 35, 9349.

3 H. Tateishi, K. Hatakeyama, C. Ogata, K. Gezuhara, J. Kuroda, A. Funatsu, M. Koinuma, T. Taniguchi, S. Hayami and Y. Matsumoto, J. Electrochem. Soc., 2013, 160, F1175; K. Hatakeyama, M. R. Karim, C. Ogata, H. Tateishi, A. Funatsu, T. Taniguchi, M. Koinuma, S. Hayami and Y. Matsumoto, Angew. Chem., Int. Ed., 2014, 53, 6997.

4 K. Raidongia and J. Huang, J. Am. Chem. Soc., 2012, 134, 16528.

5 R. kumar and K. Scott, Chem. Commun., 2012, 48, 5584; H. Zarrin, D. Higgins, Y. Jun, Z. W. Chen and M. Fowler, J. Phys. Chem. C, 2011, 115, 20774; Y. He, J. Wang, J. Liu, H. Zhang, T. Zhang, B. Zhang and S. Cao, J. Mater. Chem. A, 2014, 2, 9548.

6 M. R. Karim, K. Hatakeyama, T. Matsui, H. Takehira, T. Taniguchi, M. Koinuma, Y. Matsumoto, T. Akutagawa, T. Nakamura, S. Noro, T. Yamada, H. Kitagawa and S. Hayami, J. Am. Chem. Soc., 2013, 135, 8097.

7 W. Gao, G. Wu, M. T. Janicke, D. A. Cullen, R. Mukundan, J. K. Baldwin, E. L. Brosha, C. Galande, P. M. Ajayan, K. L. More, A. M. Dattelbaum and P. Zelenay, Angew. Chem., Int. Ed., 2014, 53, 3588.

8 Y. Ikeda, M. R. Karim, H. Takehira, T. Matsui, K. Hatakeyama, Y. Murashima, T. Taniguchi, M. Koinuma, M. Nakamura, Y. Matsumoto and S. Hayami, Bull. Chem. Soc. Jpn., 2014, 87, 63.

9 M. R. Karim, M. S. Islam, K. Hatakeyama, M. Nakamura, R. Ohtani, M. Koinuma and S. Hayami, J. Phys. Chem. C, 2016, 120, 21976.

10 M. S. Islam, M. R. Karim, K. Hatakeyama, H. Takehira, R. Ohtani, M. Nakamura, M. Koinuma and S. Hayami, Chem.-Asian J., 2016, 11, 2322.

11 K. Hatakeyama, M. R. Karim, C. Ogata, H. Tateishi, T. Taniguchi, M. Koinuma, S. Hayami and Y. Matsumoto, Chem. Commun., 2014, 50, 14527.

12 J. Wang, L. Zhao, D. Wei, W. Wu, J. Zhang and X. Cheng, Ind. Eng. Chem. Res., 2016, 55, 11931.

13 Y. Ikeda, M. R. Karim, H. Takehira, T. Matsui, K. Hatakeyama, Y. Murashima, T. Taniguchi, M. Koinuma, M. Nakamura, Y. Matsumoto and S. Hayami, Bull. Chem. Soc. Jpn., 2014, 87, 639.

14 G. Bekiaris, C. Peltre, L. S. Jensen and S. Bruun, Spectrochim. Acta, Part A, 2016, 168, 29; G. Socrates, Infrared and Raman Characteristic Group Frequencies: Tables and Charts, John Wily \& Sons Ltd., Chichester, England, 3rd edn, 2001.

15 Y. Aoki, H. Habazaki, S. Nagata, A. Nakao, T. Kunitake and S. Yamaguchi, J. Am. Chem. Soc., 2011, 133, 3471.

16 K. Wakata, M. R. Karim, M. S. Islam, R. Ohtani, M. Nakamura, M. Koinuma and S. Hayami, Chem.-Asian J., 2017, 12, 194. 